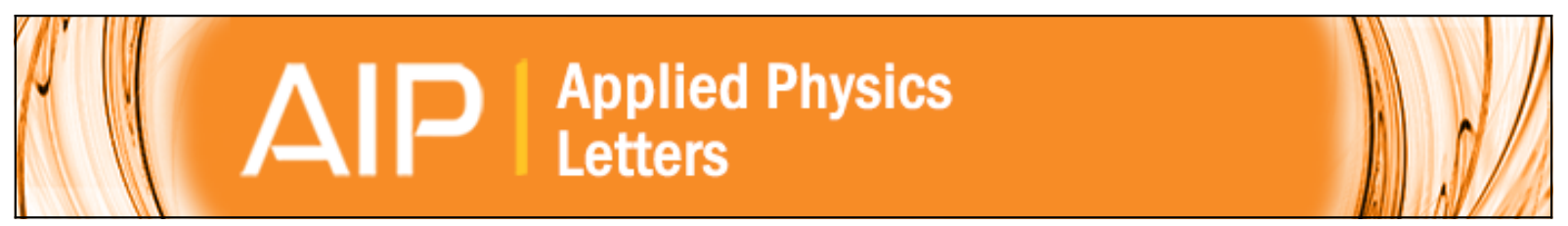

\title{
Zero displacement microelectromechanical force sensor using feedback control
}

M. Bulut Coskun, Steven Moore, S. O. Reza Moheimani, Adrian Neild, and Tuncay Alan

Citation: Applied Physics Letters 104, 153502 (2014); doi: 10.1063/1.4871380

View online: http://dx.doi.org/10.1063/1.4871380

View Table of Contents: http://scitation.aip.org/content/aip/journal/apl/104/15?ver=pdfcov

Published by the AIP Publishing

\section{Articles you may be interested in}

Nanoscale displacement sensing using microfabricated variable-inductance planar coils

Appl. Phys. Lett. 103, 143501 (2013); 10.1063/1.4823828

Note: Helical nanobelt force sensors

Rev. Sci. Instrum. 83, 126102 (2012); 10.1063/1.4769757

Fast on-wafer electrical, mechanical, and electromechanical characterization of piezoresistive cantilever force sensors

Rev. Sci. Instrum. 83, 015002 (2012); 10.1063/1.3673603

Micromachined force sensors for the study of cell mechanics

Rev. Sci. Instrum. 76, 044301 (2005); 10.1063/1.1863792

Force based displacement measurement in micromechanical devices

Appl. Phys. Lett. 78, 4031 (2001); 10.1063/1.1380398

Frustrated by

old technology?

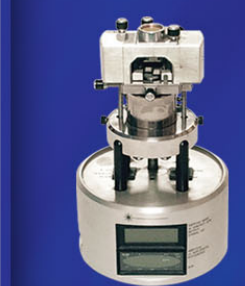

Is your AFM dead

and can't be repaired?

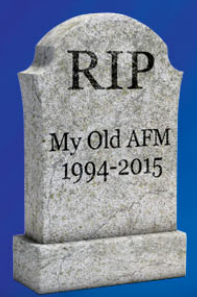

Sick of bad customer support?

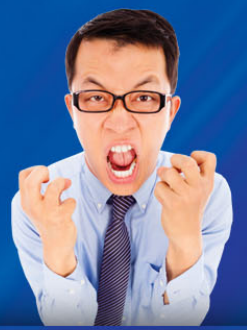

It is time to upgrade your AFM Minimum $\$ 20,000$ trade-in discount for purchases before August 31st

Asylum Research is today's technology leader in AFM

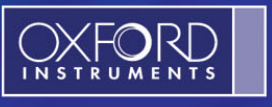




\title{
Zero displacement microelectromechanical force sensor using feedback control
}

\author{
M. Bulut Coskun, ${ }^{1}$ Steven Moore,${ }^{2}$ S. O. Reza Moheimani, ${ }^{2}$ Adrian Neild, ${ }^{1}$ \\ and Tuncay Alan ${ }^{1, a)}$ \\ ${ }^{1}$ Laboratory for Micro Systems, Department of Mechanical and Aerospace Engineering, Monash University, \\ Melbourne, VIC 3800, Australia \\ ${ }^{2}$ Laboratory for Dynamics and Control of NanoSystems (LDCN), School of Electrical Engineering \\ and Computer Science, University of Newcastle, Callaghan, NSW 2308, Australia
}

(Received 11 March 2014; accepted 3 April 2014; published online 14 April 2014)

\begin{abstract}
Conventional microscale force sensors use moving parts to infer applied forces. Whenever physical deformations are involved, the sensor characteristics become a function of mechanical parameters, and there is an inevitable trade-off between the sensitivity and measurement range. We developed a microfabricated force sensor that uses feedback control to nullify any displacements within the device, directly transducing forces as high as $1.5 \mathrm{mN}$ with a $7.8 \mathrm{nN}$ resolution. The range and sensitivity of the device no longer depend on mechanical parameters, which allow the same device to be used to test samples with a wide range of stiffnesses without loss of accuracy. (C) 2014 AIP Publishing LLC. [http://dx.doi.org/10.1063/1.4871380]
\end{abstract}

Accurate quantification of mechanical forces and displacements at micro/nano size scales is crucial for a myriad of applications, including characterization of biological tissues $^{1-3}$ and cells, ${ }^{4-6}$ microgripping/manipulation ${ }^{7,8}$ of particulate matter, and mechanical reliability of nanoscale structures $^{9}$ to name a few. Silicon based microelectromechanical systems (MEMS) have received a significant attention as miniaturized force sensors due to their high sensitivity and customizability as well as the availability of cost-effective batch fabrication techniques. Typically, a MEMS force sensor consists of mechanical sensory parts (such as a shuttle $e^{10}$ or deformable structures ${ }^{11}$ ) that move under applied forces and the corresponding circuitry transducing this mechanical displacement into an electrical signal. The force values can then be determined taking into account the detected displacements and the mechanical stiffness of the device.

This conventional approach has several drawbacks: First, when the deformations are involved, the range and sensitivity of the sensor become a function of mechanical parameters. There is an inevitable trade-off between the sensitivity and measurement range where the stiffness of the sensor plays the most important role in the sensor performance. Low stiffness systems provide high sensitivity accompanied with a low sensing range, whereas higher stiffnesses result in a greater sensing range while reducing the sensitivity. Moreover, the stiffness of the deformed sensing element also becomes displacement dependant at large deformations, hence, leading to nonlinearity in sensor response. Second, sensors with moving parts are not adequate in applications requiring high level of dexterity and manipulation accuracy. Unavoidable slacks in the sensory element could be detrimental when handling delicate biological samples ${ }^{12}$ or characterising dynamic response of rate sensitive materials. ${ }^{2}$ Whilst micro sensors with force feedback can be implemented to partially overcome this latter limitation, ${ }^{13}$ feedback control alone is not sufficient to eliminate the

a)Email: tuncay.alan@monash.edu substantial effect of the sensor stiffness on measurement range/accuracy.

To address these issues, we developed a MEMS force sensor that uses feedback control to nullify any mechanical motion within the device, directly transducing forces without incurring any displacements. Despite being widely used in accelerometers, ${ }^{14}$ force sensor studies employing "forcebalancing" with feedback control are limited in the open literature. The concept is to negate displacement of the sensor by using a feedback signal to actuate a second set of transduction elements in order to balance the applied force. Some earlier studies demonstrated this approach using centimeter scale cantilevers with embedded piezoelectric actuating and sensing layers (on the top and bottom surface, respectively), ${ }^{15}$ and others have investigated applicability of a comb drive system for increased dynamic range of force measurement. ${ }^{16}$ In this mode of operation, the range and sensitivity of the sensor become a function of actuator parameters, as opposed to mechanical properties, which allows greater freedom in the design. In addition, the need for the mechanical calibration of the force sensor is removed, hence, making the approach useful for testing different samples with varying stiffness values. Here, we demonstrate this advantage by employing the same device to calibrate commercial AFM cantilevers with stiffnesses ranging from 13.17 to $244.42 \mathrm{~N} / \mathrm{m}$ without loss of accuracy. Other potential advantages of the developed system are in applications, in which a defined strain-rate is required.

The device was fabricated through a commercial 3mask silicon-on-insulator multi-user MEMS process (SOIMUMPS). ${ }^{17}$ The sensing components consist of compact ( $50 \mu \mathrm{m} \times 25 \mu \mathrm{m} \times 25 \mu \mathrm{m})$ electro-thermal displacement sensors ${ }^{18}$ positioned on the sides of a moveable $(2.6 \mathrm{~mm} \times 2 \mathrm{~mm}$ wide and $25 \mu \mathrm{m}$ thick) shuttle mass (Fig. 1(a)). The shuttle is supported by 64 slender flexures $(1100 \mu \mathrm{m} \times 3.5 \mu \mathrm{m})$ to minimize the torsional effects and out-of-plane deflections. Importantly, the device also incorporates three sets of electrostatic comb-drive actuators (Fig. 1(b)) which are used to balance the external forces detected by the sensors. 

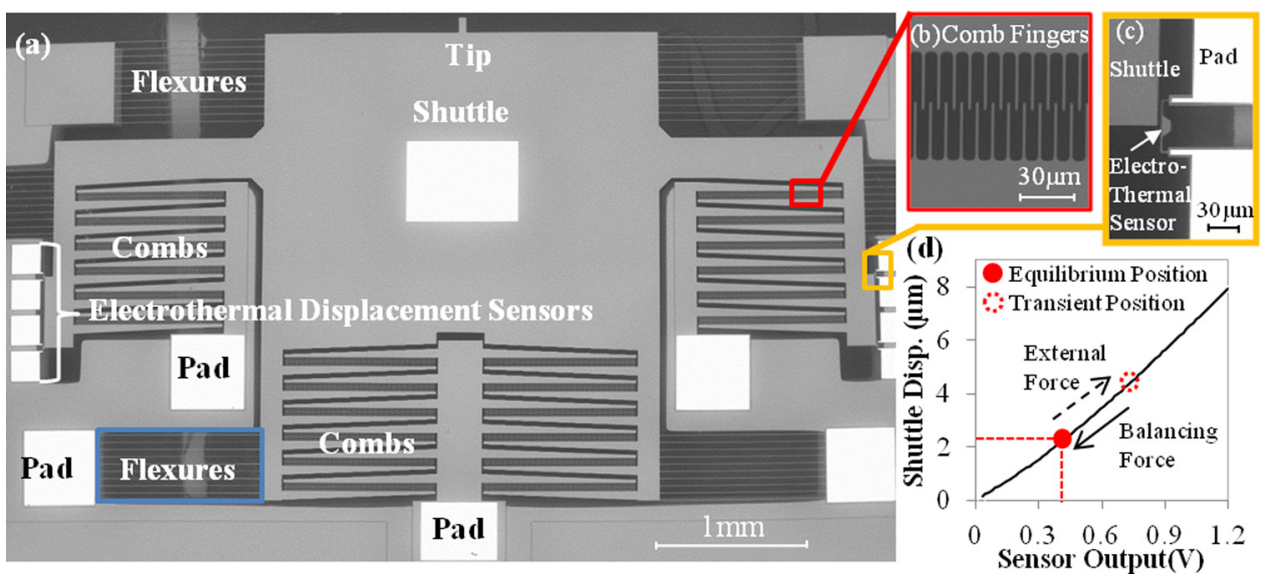

FIG. 1. (a-c) Design and components of the MEMS force sensor (contrast enhanced). (d) Relation between displacement of the shuttle and the corresponding electro-thermal displacement sensor voltage to the comb drives. The transient displacement of the shuttle due to an external force is nullified by the balancing force that is generated by the comb drive according to the feedback signal.

The comb drives are controlled by an external feedback control circuit and ensure that the position of the shuttle is held fixed at an equilibrium position, regardless of the applied external forces. The large number of comb-actuators allows a wide force range (maximum force of $1.5 \mathrm{mN}$ ), whereas the compact electro-thermal sensing elements (Fig. 1(c)) allow the form factor to be small (the entire device fits onto a $3.5 \times 8 \mathrm{~mm}^{2}$ chip).

Prior to force application, a $10 \mathrm{~V}$ bias voltage applied to the comb-drives displaces the shuttle by $2.07 \mu \mathrm{m}$. This initial offset ensures that all voltages throughout the operation are positive and in doing so minimizes the instabilities. Throughout the operation, when the control action is in place, an external force that leads to a transient displacement of the shuttle is compensated by the force generated by the combdrives (Fig. 1(d)). Hence, the shuttle remains at the equilibrium position of $2.07 \mu \mathrm{m}$, regardless of the external forces.

Fig. 2(a) depicts the frequency response data, which is used to identify the open loop transfer function of the system from the input voltage to the electro-thermal sensor output. The measurement shows that the system is of 2 nd order with a resonant frequency of $1.497 \mathrm{kHz}$. When an external force is applied to the tip, displacement of the shuttle leads to a change in the heat flow from the shuttle to the electrothermal displacement sensor. The temperature variations of the resistive heaters alter the resistance that can be read out electrically. In the control loop, the input stimulus to the sensor appears as an input disturbance, " $f$ " (Fig. 2(b)). Using the feedback signal from the sensory part, a corresponding actuation voltage is then applied to the comb-drive which in return produces the required electrostatic force to compensate the applied external force, thus, the shuttle is moved back to the equilibrium position. Therefore, the control action " $V$ " compensates for the input disturbance, and becomes the output of the sensor. The complete control topology is shown in Fig. 2(b). The bandwidth of the system is acquired from the complementary sensitivity function, $T$, of the closed loop system that maps the reference " $r$ " (Fig. 2(c)) to the electro-thermal sensor output. By setting $r=0$, the controller will act to keep the stage in its equilibrium position. The controller can perform reference tracking up to
$70 \mathrm{~Hz}$. Hence, minimum response time of the system is estimated to be $14 \mathrm{~ms}$.

This operation principle lends interesting advantages to the measurement of AFM cantilevers. A schematic of the experiments to demonstrate this is shown in Fig. 3(a). The cantilevers were fixed on a custom-built holder and the force sensor/control circuit assembly was mounted on a motorized stage (Newport M-562-XYZ-LH) actuated by a step motor (NewFocus 8310 picomotor driven by $8751-\mathrm{CL}$ closed-loop picomotor driver). After contact was established with the fixed AFM cantilever tip, the sensor was pushed against the cantilever in $600 \mathrm{~nm}$ steps deforming it (Fig. 3(a)) in the out

(a)

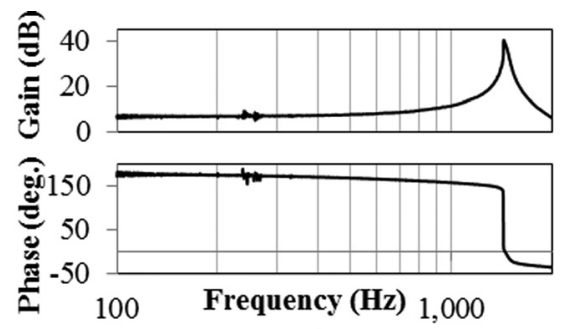

(b)

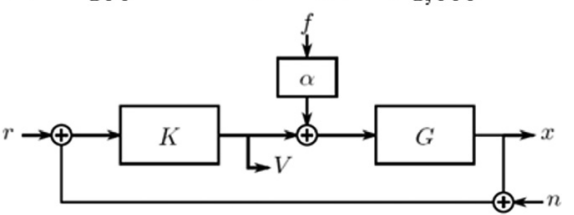

(c)

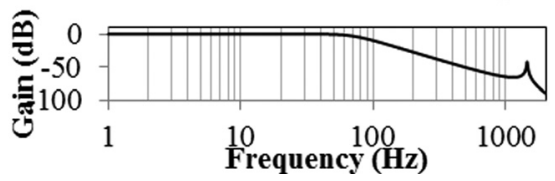

FIG. 2. (a) The frequency response of the device obtained by measuring the electro-thermal sensor output as a function of the actuation voltage. (b) The topology of the control system. $G$ is the MEMS device with a voltage input and electro-thermal sensor output $x$. The controller $K$ is designed for disturbance rejection with the reference $r=0$. As a sensor, the input disturbance $\mathrm{f}$ is the input stimulus and the value of the control action $\mathrm{V}$ is the output reading. Of consideration is the effect of the electro-thermal sensor noise $n$ on the force sensor performance. (c) The complementary sensitivity function $T$ of the system. It is the mapping from the reference $r$ to the electro-thermal sensor output $x$. The control system has reference tracking up to a bandwidth of $70 \mathrm{~Hz}$. 

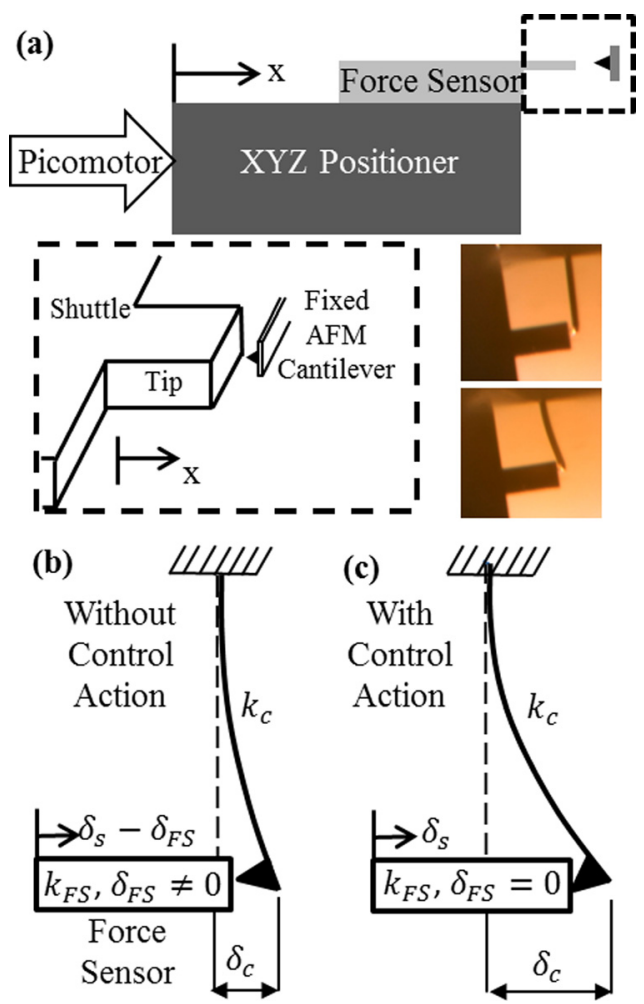

FIG. 3. (a) A schematic view of the test setup and optical micrographs showing the force sensor deflecting AFM cantilevers. Diagram depicting the displacement relations of the AFM cantilevers without (b) and with (c) the feedback control that nullifies the sensor displacement.

of plane direction. Before proceeding with the results, it is useful to briefly discuss the behavior of the sensor with and without the control loop.

In the absence of control action (Fig. 3(b)), when the stage is displaced by $\delta_{s}$, the cantilever would be deflected in the same direction by $\delta_{c}$ under an applied force $F_{c}$. Similarly, the movable shuttle tip would displace in the opposite direction by $\delta_{F S}$ under an equal magnitude reaction force. The relation between the displacements and forces are as follows:

$$
\begin{gathered}
\delta_{c}=\delta_{s}-\delta_{F S}, \\
F_{c}=k_{c} \delta_{c}=-k_{F S} \delta_{F S},
\end{gathered}
$$

where $k_{F S}$ is the sensor stiffness, provided by the 64 flexures, and $k_{c}$ is the stiffness of the tested cantilever. The magnitude of $\delta_{s}$ is known since this is the displacement of the stage introduced to the system by the picomotor (Fig. 3(a)). However, in the absence of feedback control either $\delta_{F S}$ or $\delta_{c}$ have to be measured, and $k_{F S}$ must be determined. Displacements can be optically measured, however, measurement of $k_{F S}$ requires further characterization steps, and is usually acquired separately by externally calibrated sensors ${ }^{19}$ or using reference cantilevers with known stiffnesses. ${ }^{20}$ Higher number of intermediate steps increases the cumulative uncertainty in the final output of the sensor.

Additionally, the stiffness of the shuttle, $k_{F S}$ also substantially affects the sensitivity and sensing range of the sensor. Due to previously mentioned sensitivity-range trade-off, for best precision, the sensor stiffness should be comparable to that of the test sample. Since the deformation is allowed, large deflections result in nonlinear behavior due to stiffening of the flexures that also limits the measurement range. The schematic explanation without the control action is shown in Fig. 3(b).

When the control is used (Fig. 3(c)), the shuttle tip is kept steady at the equilibrium position through an electrostatic force, $F_{e s}$, applied by the comb-drives, thus, $\delta_{F S}$ is zero throughout the loading and the stage movement becomes directly equal to the cantilever deflection $\left(\delta_{c}=\delta_{s}\right)$. Hence, the force applied on the cantilever can be expressed as $F_{c}=k_{c} \delta_{c}=k_{c} \delta_{s}$. Since the electrostatic force, $F_{e s}$, generated by the comb drive to nullify the displacement is equal to $F_{C}$, the applied force is then given as

$$
F_{c}=F_{e s}(V)=\frac{1}{2} \frac{\partial C(x)}{\partial x}\left(V_{\text {in }}^{2}-V_{\text {bias }}^{2}\right)=k_{c} \delta_{s},
$$

where, $\frac{\partial C}{\partial x}$ is the rate of change of capacitance of the combdrives with respect to the position, $x$ and is a constant for the system (calculated through finite element analysis (FEA) simulation, including the effect of fringe fields by using the actual dimensions of the comb drives). The other values are known inputs, $V_{\text {bias }}$ is the bias voltage $(10 \mathrm{~V}$ initially to keep the stage at the equilibrium position when no force is acting on the sensor tip) and $V_{\text {in }}$ is a function of the sensor output, the only variable corresponding to the net force acting on the shuttle. (The maximum operating voltage for the combdrives is set to $50 \mathrm{~V}$, in our experiments, after a gain of 20 is applied to the actuation voltage).

When an external force is applied, the transient displacement of the shuttle increases the sensor voltage (Fig. 4(a)). Simultaneously, control action produces a voltage applied to the combs ( $V_{\text {bias }}$ is increased to $V_{\text {in }}$ ) to cancel the displacement of the shuttle (Fig. 4(b)) and as a result, the sensor voltage reduces back to zero. Clearly, the transient response of the device depends on the stiffness of the sensor. For the particular case of Fig. 4, by keeping other parameters the same but decreasing the sensor stiffness, one can expect larger changes in sensor and actuation voltages. However, since the system does not allow variation in the stiffness by nullifying the deformation, the overall effect of sensor stiffness to

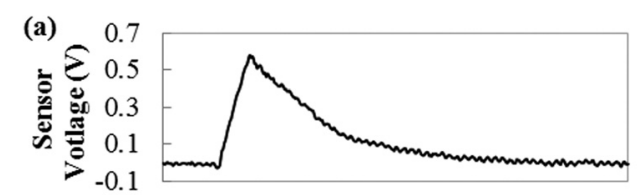

(b)

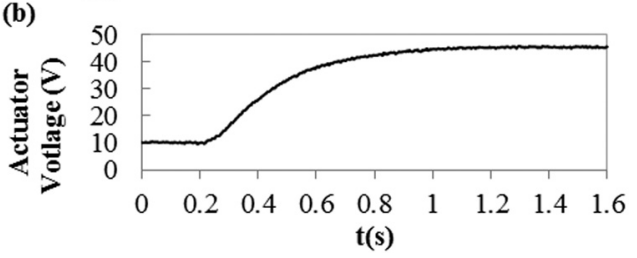

FIG. 4. Step response of the system when the device is pushed against the cantilever. (a) As the stage moves the heat conducted into the stage increases along with the temperature. Resistance of the heaters varies accordingly to produce a voltage output. As the stage moves back to equilibrium, sensor voltage returns to zero. (b) Input voltage increases to balance the external force and bring the shuttle back to the equilibrium. 


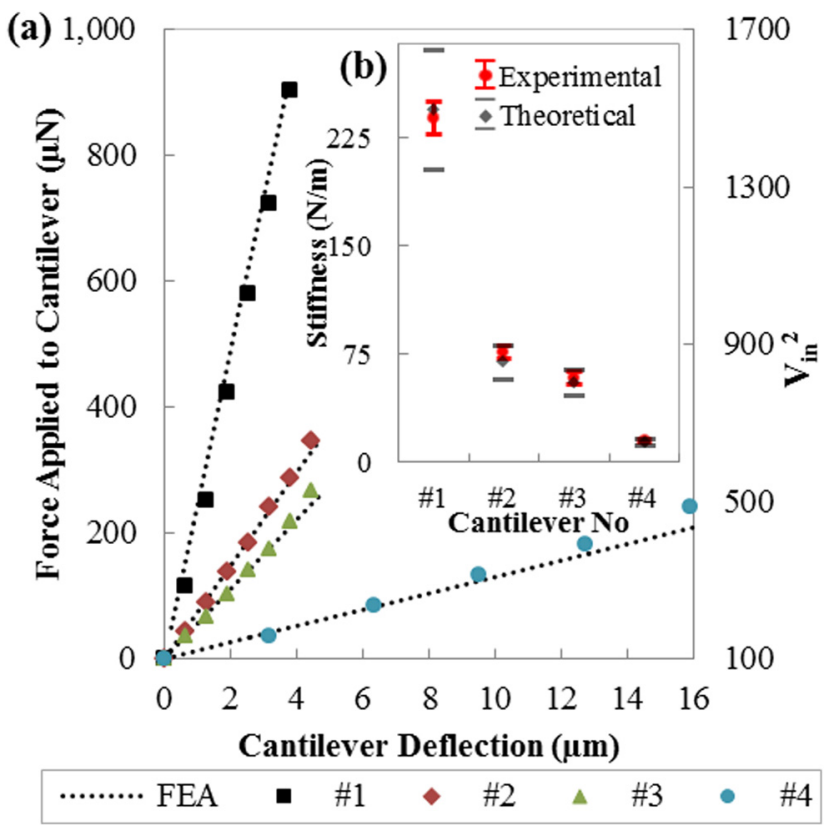

FIG. 5. (a) Comparison of experimental and theoretical force data for the four AFM cantilevers. (b) Comparison of the measured and calculated stiffness values. The experimental uncertainty is $4.24 \%, 5.80 \%, 6.74 \%$, and $6.61 \%$, for cantilevers \#1-4 respectively. The uncertainty in the theoretical calculation is $16.8 \% .^{20}$

measurement accuracy is significantly less than that in the open-loop configuration.

Returning back to the experiments; we used a single force sensor to characterize mechanical properties of four different AFM cantilevers $(1 \times$ VeecoTAP525, $2 \times$ VEECO TESPA, and $1 \times \mathrm{NSG01)}$ with calculated ${ }^{20}$ stiffnesses of $244.42 \mathrm{~N} / \mathrm{m}, 69.57 \mathrm{~N} / \mathrm{m}, 55.44 \mathrm{~N} / \mathrm{m}$, and $13.17 \mathrm{~N} / \mathrm{m}$, respectively, using the setup in Fig. 3(a). All of the tests were repeated four times monitoring $V_{\text {in }}$ generated by the combs to nullify the sensor displacement and directly obtaining the corresponding forces using Eq. (3). Similarly, the cantilever deflection $\delta_{c}$ was obtained by measuring $\delta_{s}$ throughout the loading. Experimentally, the minimum force we observed was $4.4 \mu \mathrm{N}$ within a range of $0-1500 \mu \mathrm{N}$ due to the limited resolution of the measurement equipment. However, evaluation of the noise floor ${ }^{21}$ indicates that resolution of the sensor within the $70 \mathrm{~Hz}$ bandwidth is $7.8 \mathrm{nN}$.

Fig. 5 shows that there is a good agreement between the experimental load deflection curves and the theoretical predictions obtained through a 3D FEA. ${ }^{20}$ The inset, Fig. 5(b) presents the measured and calculated stiffness values with the corresponding uncertainties (estimated ${ }^{22}$ considering Eq. (3) and taking into account any measurement uncertainties in voltage read-out as well as geometric parameters). Relative uncertainties in stiffness measurements $(4.24 \%$, $5.80 \%, 6.74 \%$, and $6.61 \%$ for the cantilevers 1 to 4 , respectively) are comparable to conventional experimental methods $\left(7.9 \%\right.$ through reference cantilever method, ${ }^{20} 5 \%$ for the resonant frequency analysis ${ }^{23}$ and for thermal tuning ${ }^{24}$ ). However, the force balancing approach presented here has a significantly higher range and is relatively insensitive to cantilever geometry and shape.

In conclusion, a series of experiments were conducted to demonstrate the performance of the force sensor based on force balancing principle. It is shown that the device can handle samples with a wide range of stiffnesses without loss of the sensitivity. Hence, the trade-off between sensitivity and sensing range is minimized together with the sensor calibration effort and complexity of the sample characterization.

Part of this work was performed at the Melbourne Centre for Nanofabrication (MCN) in the Victorian Node of the Australian National Fabrication Facility (ANFF).

${ }^{1}$ Y. Zhao and X. Zhang, Appl. Phys. Lett. 87, 144101 (2005).

${ }^{2}$ P. Du, X. Zheng, I. Lin, and X. Zhang, Appl. Phys. Lett. 99, 083701 (2011).

${ }^{3}$ J. Rajagopalan, A. Tofangchi, and M. T. A. Saif, J. Microelectromech. Syst. 19, 1380 (2010).

${ }^{4}$ Y. Mao, Q. Sun, X. Wang, Q. Ouyang, L. Han, L. Jiang, and D. Han, Appl. Phys. Lett. 95, 013704 (2009).

${ }^{5}$ D. H. Kim, Y. Sun, S. Yun, S. H. Lee, and B. Kim, J. Biomech. 38, 1359 (2005).

${ }^{6}$ I. Sokolov, S. Iyer, V. Subba-Rao, R. M. Gaikwad, and C. D. Woodworth, Appl. Phys. Lett. 91, 023902 (2007).

${ }^{7}$ P. J. Berkelman, L. L. Whitcomb, R. H. Taylor, and P. Jensen, IEEE Trans. Rob. Autom. 19(5), 917 (2003).

${ }^{8}$ F. Beyeler, A. Neild, S. Oberti, D. J. Bell, Y. Sun, J. Dual, and B. J. Nelson, J. Microelectromech. Syst. 16, 7 (2007).

${ }^{9}$ T. Alan, A. T. Zehnder, D. Sengupta, and M. A. Hines, Appl. Phys. Lett. 89, 231905 (2006).

${ }^{10}$ S. J. Koch, G. E. Thayer, A. D. Corwing, and M. P. de Boer, Appl. Phys. Lett. 89, 173901 (2006).

${ }^{11}$ T. Chu Duc, J. F. Creemer, and P. M. Sarro, IEEE Sens. J. 7, 96 (2007).

${ }^{12}$ Z. Lu, P. C. Y. Chen, and W. Lin, IEEE Trans. Syst. Man Cybern., Part C Humans. 36, 713 (2006).

${ }^{13}$ K. Kim, X. Liu, Y. Zhang, and Y. Sun, J. Micromech. Microeng. 18, 055013(2008).

${ }^{14}$ H. Luo, Z. Gang, L. R. Carley, and G. K. Fedder, J. Microelectromech. Syst. 11, 188 (2002)

${ }^{15}$ Y. Shen, E. Winder, X. Ning, C. A. Pomeroy, and U. C. Wejinya, IEEE/ASME Trans. Mechatronics 11, 420 (2006).

${ }^{16}$ Y. Sun, D. P. Potasek, D. Piyabongkarn, R. Rajamani, and B. J. Nelson, in Proceedings of ICRA (2003), p. 29.

${ }^{17}$ A. Cowen, G. Hames, D. Monk, S. Wilcenski, and B. Hardy, SOIMUMPS Design Handbook, Revision 6.0 (MEMSCAP, Inc., 2009).

${ }^{18}$ A. G. Fowler, A. Bazaei, and S. O. R. Moheimani, J. Microelectromech. Syst. 22, 687 (2013).

${ }^{19}$ F. Beyeler, S. Muntwyler, and B. J. Nelson, J. Microelectromech. Syst. 18, 433 (2009).

${ }^{20}$ S. J. Grutzik, R. S. Gates, Y. B. Gerbig, D. T. Smith, R. F. Cook, and A. T. Zehnder, Rev. Sci. Instrum. 84, 113706 (2013).

${ }^{21}$ A. J. Fleming, Sens. Actuators, A 190, 106 (2013).

${ }^{22}$ H. H. Ku, "Notes on the Use of Propagation of Error Formulas," J. Res. Natl. Bur. Stand. 70C, 263 (1966). Available at http://nistdigitalarchives. contentdm.oclc.org/cdm/compoundobject/collection/p13011 coll6/id/78003/ $\mathrm{rec} / 5$.

${ }^{23}$ J. E. Sader, I. Larson, P. Mulvaney, and L. R. White, Rev. Sci. Instrum. 66, 3789 (1995).

${ }^{24}$ B. Ohler, Rev. Sci. Instrum. 78, 063701 (2007). 